\title{
Observation of isothermal metamorphism of new snow and interpretation as a sintering process
}

\author{
T. U. Kaempfer ${ }^{1,2}$ and M. Schneebeli ${ }^{1}$ \\ Received 6 June 2007; revised 20 August 2007; accepted 11 September 2007; published 20 December 2007.
}

[1] Isothermal snow metamorphism is based on the fundamental process of sintering. However, the relative weights of the physical processes responsible for sintering between ice grains are still debated. The most active are expected to be grain boundary diffusion or sublimation-condensation. We performed four isothermal experiments, starting with fresh snow, at temperatures of $-1.6,-8.3,-19.1$, and $-54{ }^{\circ} \mathrm{C}$ during nearly 1 year. Monthly, we imaged the snow samples by X-ray microtomography. We monitored the changes in density, specific surface area, structure model index, and several structural parameters based on the distance transform of the ice matrix and pore space in the snow, as the trabecular number and thickness. At $-54{ }^{\circ} \mathrm{C}$, the metamorphism process was very slow, and during 1 year the specific surface area decreased by only $19 \%$. For the other samples, the results can be interpreted as a two-stage process with a first phase of rapid change in trabecular number and structure model index and a second phase where the trabecular number and structure model index were constant. An increase in density and trabecular thickness together with a decrease of the specific surface area following a power law were observed throughout the experiments. The increase in ice thickness (coarsening) related linearly to the densification. The continuous densification implies that volume processes like grain boundary diffusion have to occur. The linear relation between densification and coarsening suggests that the same mechanism governs both processes. The logarithmic decrease of the specific surface area is an indication that the coarsening is rate limiting.

Citation: Kaempfer, T. U., and M. Schneebeli (2007), Observation of isothermal metamorphism of new snow and interpretation as a sintering process, J. Geophys. Res., 112, D24101, doi:10.1029/2007JD009047.

\section{Introduction}

[2] Snow is a porous sintered material consisting of ice, air, small amounts of impurities, and sometimes liquid water. The ice grains are mostly monocrystalline and are connected by bonds. Sintering and associated changes in the microstructural shape of the snow are caused by high temperatures close to the melting point of water, always the case under terrestrial conditions. This process is known as snow metamorphism. Metamorphism is described as dry and isothermal if no temperature gradient exists and the temperature is below the melting point of ice. In this case, diffusional water vapor transport in the pore space is solely driven by Kelvin effects. Conditions for long-term isothermal metamorphism occur in nature only in the center of the polar ice shields [Arnaud et al., 1998].

[3] The metamorphosing snow microstructures directly influence snowpack properties, including mechanical properties used in avalanche forecasting [Schweizer et al., 2003],

\footnotetext{
${ }^{1}$ WSL Swiss Federal Institute for Snow and Avalanche Research, Davos, Switzerland.

${ }^{2}$ Now at Cold Regions Research and Engineering Laboratory, Hanover, New Hampshire, USA.

Copyright 2007 by the American Geophysical Union. 0148-0227/07/2007JD009047
}

chemical composition associated with the interpretation of ice cores data [Legrand and Mayewski, 1997], and thermophysical properties important for modeling the energy balance of snow-covered landscapes [Sokratov and Barry, 2002a, 2002b]. These properties evolve thus simultaneously with the snow microstructure during metamorphism. In order to improve the various snow cover models it is necessary to understand the physical processes of snow metamorphism and deduce relevant parameters. Some snow properties depend primarily on the grains, such as the optical properties, where scattering and absorption depend on grain size and absorptive impurities [Warren, 1982]. Other properties are most sensitive to the bonds between grains, where mechanical or thermal stresses are larger, as for example the cohesion stress which can be related to the bond surface [Shapiro et al., 1997]. Thermal conductivity is also strongly dependent on the microstructure [Arons and Colbeck, 1995] and has been simulated for a natural snow structure [Kaempfer et al., 2005]. Finally, processes like snow-air interaction might depend on parameters like the specific surface area (SSA) of the ice matrix, i.e., the surface area of snow accessible to gases per unit volume [Dominé and Shepson, 2002].

[4] While snow metamorphism in natural snowpacks occurs mostly under varying conditions, including changing temperature gradients or the presence of a liquid phase, it is 
important to reduce the physical complexity of experiments in order to gain insight into the processes governing metamorphism. We thus limit this work to isothermal metamorphism in dry snow and we refer to the review by Colbeck [1997] and the references therein for an overview on the different forms of metamorphism.

[5] Isothermal metamorphism of snow is a sintering process between the ice grains forming the snow. Under the absence of temperature gradients, the driving force for snow metamorphism is the sintering stress, which tends to reduce the surface free energy of the complex ice-air interfaces and of the grain boundaries between the ice crystals. The energy reduction of the porous structure is achieved by mass redistribution. Mass transport processes include surface diffusion, volume diffusion, grain boundary diffusion, viscous flow, plastic flow, and evaporation-condensation with vapor transport [German, 1996; Maeno and Ebinuma, 1983]. In sintering theories, the common approach consists in deriving a model growth law of the form

$$
\left(\frac{X}{D}\right)^{n}=\frac{B t}{D^{m}},
$$

where $X$ is the grain bond diameter, $D$ is the particle diameter, $t$ is the isothermal sintering time, and $B$ is a term that collects material and geometric properties. Then, from the experimentally determined exponents $n$ and $m$, as well as from $B$, one tries to determine the dominant mass transport process [German, 1996]. For example, values of $n=7$ and $m=4$ would indicate surface diffusion, $n=5$ and $m=3$ volume diffusion, and $n=3$ and $m=2$ would suggest that the dominant process is evaporation-condensation.

[6] Several experiments with this goal were performed for ice particles and led to different estimates of $n$ and $m$ and thus to different conclusions. Kingery [1960] concluded that surface diffusion was the dominant process. However, his coefficient of surface diffusion required was very high, which could be explained by a highly mobile surface transition layer. The experiments of Kuroiwa [1961] were interpreted as that volume diffusion was responsible for the sintering of ice, while Hobbs and Mason [1963] concluded that sublimation-condensation and transport through the vapor phase had to be the dominant process. The vapor transfer mechanism has since received wide acceptance. Flin et al. [2003] developed a numerical model of curvature-dependent snow metamorphism on the microstructural level and compared the computational results to tomographic data of a natural snow sample undergoing isothermal metamorphism at $-2^{\circ} \mathrm{C}$ for three months. Flin et al. [2004] used the same experimental data set to compute the evolution of structural parameters like the SSA, anisotropy, porosity, and curvature. However, they noted that no conclusion could be made regarding the validity of the evaporation-condensation approach. In his most recent works, Colbeck [1997, 1998, 2001] points out that for the treatment of snow metamorphism by vapor transfer the wrong geometry has been assumed. In fact, as ice is a crystalline material, a boundary groove will form at the grain boundary between two adjacent ice grains. This was confirmed by several observations of grain boundaries, but not always interpreted [Kuroiwa, 1962; Colbeck, 1997; Legagneux et al., 2003]. Thus all the models using a concave bond geometry and associated transport from convex grains to the concave bond cannot be valid as soon as the grain boundary groove has formed. Colbeck [1998, 2001] proposes grain boundary diffusion as one of the dominant processes during isothermal metamorphism.

[7] Not clear is the influence of impurities on snow metamorphism. Using scanning electron microscopy, Rosenthal et al. [2006] localized impurity concentrations near the grain boundaries in snow and from industrial sintering studies dopants are well known to alter the sintering processes.

[8] Considerable work on the evolution of the SSA of snow undergoing isothermal metamorphism has recently been conducted. Cabanes et al. [2003] showed that the SSA almost always decreases along with metamorphism and proposed an empirical equation relating the rate of decrease to temperature. Legagneux et al. [2003] performed isothermal experiments at $-4,-10$, and $-15^{\circ} \mathrm{C}$, determined the SSA decrease by methane adsorption at $77 \mathrm{~K}$ followed by the Brunauer-Emmett-Teller (BET) treatment of the adsorption isotherm, and observed the snow by scanning electron microscopy. They found that the SSA decreased logarithmically with time. The same authors conducted several isothermal experiments at $-15^{\circ} \mathrm{C}$ which confirmed the logarithmic trend [Legagneux et al., 2004]. They interpreted this decay using a grain growth theory related to Ostwald Ripening. These theories originally describe the coarsening of a distribution of sufficiently spaced spherical particles by exchange of matter through a liquid phase but have been adapted to denser packing, nonspherical particles, and diffusion through a gas phase [Legagneux and Dominé, 2005, and references therein]. In contrast, the hypothesis of nonoverlapping particles cannot be relaxed and is clearly violated in snow with its connected ice matrix. Legagneux and Dominé [2005] continued their approach by developing a mean field theory to model the SSA decrease. They implicitly assume that the dominant transport mechanism is evaporation-condensation, which contradicts observations of Colbeck [2001]. Moreover, the Kelvin equation shows that for grain radii greater than about $0.1 \mu \mathrm{m}$, the effect of the radius of curvature on saturation vapor pressure becomes very small. Nevertheless, the logarithmic trend for the SSA decrease was very recently again confirmed experimentally by Taillandier et al. [2007]. The latest physical model by Flanner and Zender [2006] to predict SSA evolution and snow albedo under isothermal or temperature gradient conditions also reproduces the logarithmic trend for the SSA decrease in the isothermal case.

[9] However, there is still a considerable lack of experimental data to confirm or disprove the different SSA evolution and sintering theories. In particular, no long-term experiments with complete data on the structural evolution and at different temperatures are available. The present work tries to fill in this gap by presenting four isothermal metamorphism experiments conducted over nearly 1 year and periodically monitored nondestructively using X-ray microtomography. All the four samples were initially prepared from the same, fresh snow, but then exposed to four different temperatures ranging from $-1.6^{\circ} \mathrm{C}$ down to $-54^{\circ} \mathrm{C}$. We monitored the evolution of the density and the SSA. We used image analysis techniques based on the 
Table 1. Experimental Conditions (Temperature $T$ and Homologous Temperature $T_{h}$ ) and CT Measurements as Days From the Beginning of the Experiments for the Four Snow Samples

\begin{tabular}{|c|c|c|c|c|c|c|c|c|c|}
\hline \multirow{2}{*}{$\begin{array}{l}\text { Sample } \\
\text { Number }\end{array}$} & \multirow[b]{2}{*}{$T,{ }^{\circ} \mathrm{C}$} & \multirow[b]{2}{*}{$T_{h}(-)$} & \multicolumn{7}{|c|}{$\begin{array}{l}\text { Measurement Number and } \\
\text { Times, days }\end{array}$} \\
\hline & & & 0 & 1 & 2 & 3 & 4 & 5 & 6 \\
\hline 1 & -1.6 & 0.99 & 0 & 34 & 76 & 118 & 162 & 211 & 317 \\
\hline 2 & -8.3 & 0.97 & 0 & 34 & 76 & 118 & 162 & 215 & 316 \\
\hline 3 & -19.1 & 0.93 & 0 & 34 & 75 & 117 & 162 & 215 & 319 \\
\hline 4 & -54.0 & 0.80 & 0 & 34 & 75 & 118 & 162 & 215 & 319 \\
\hline
\end{tabular}

distance transform to deduce thickness distributions for the ice matrix and pore space and observed their evolution. A structure model index giving information about the dominating form, ranging from plates to spheres, was also regularly computed. All the monitored structural parameters were deduced from observed three dimensional snow microstructures; they are volumetric parameters whereas other investigations often used two dimensional observations from thin sections or surfaces.

\section{Experiments}

[10] Four snow samples for isothermal metamorphism experiments at different temperatures were prepared in a similar way and from the same initial snow as follows. Fresh new snow was collected and sieved at $-20^{\circ} \mathrm{C}$ through a $1 \mathrm{~mm}$ mesh into cylindrical sample holders with a diameter of $18.5 \mathrm{~mm}$ and a height of $65 \mathrm{~mm}$. The sieving was done such that the rate of filling was almost constant, which can be done manually for small samples as used here. Once filled, the sample holders were sealed with tape in order to prevent sublimation.

[11] During the whole experiment, except for the short times when the samples were measured, each sample resided inside a temperature controlled environment. A box with $10 \mathrm{~cm}$ thick walls of Styrofoam was kept in a temperature controlled cold room. The sample cylinders resided inside a steel cylinder of $10 \mathrm{~cm}$ diameter and $1 \mathrm{~cm}$ thick walls, capped with $1 \mathrm{~cm}$ thick steel lids. This cylinder eliminated any remaining temperature fluctuation because of its high thermal capacity and high thermal conductivity. A numerical simulation of this setup showed that a daily temperature variation of $2 \mathrm{~K}$ in the cold room would result in a change of less than $0.2 \mathrm{~K}$ at the inner wall of the steel cylinder. The temperatures inside the steel cylinders were recorded by 3 temperature sensors (iButton DS1922L) per storage box. These sensors have an average accuracy of $\pm 0.5^{\circ} \mathrm{C}$. Recorded temperatures were almost constant with mean values and standard deviations of $-1.6 \pm 0.20^{\circ} \mathrm{C}$, $-8.3 \pm 0.33^{\circ} \mathrm{C}$, and $-19.1 \pm 0.09^{\circ} \mathrm{C}$ for samples 1,2 , and 3 . Because of the low temperature, continuous temperature measurements for the fourth sample failed. However, the cooling device, a commercial freezer inside a cold room, was regulated to a temperature of $-50^{\circ} \mathrm{C}$ and control measurements with a thermocouple indicated a temperature of about $-54^{\circ} \mathrm{C}$ for this sample and a daily temperature variation of less than $\pm 1{ }^{\circ} \mathrm{C}$ outside the storage box. Note that the temperatures were selected such that the homologous temperature, $T_{h}$, decreased from 0.99 to 0.93 for the first three values, and to 0.80 for the lowest, where the liquid-like surface layer on ice crystals has disappeared and the temperature is below the point of homogeneous nucleation. The key properties of the experimental setup for the four samples are summarized in Table 1.

[12] At the beginning of the experiment and roughly every month during 1 year, each sample was analyzed as follows. The sample was weighted and the snow height in the sample holder measured in order to determine the density of the snow. Knowing the weight allowed also for an estimation of the mass loss from sublimation. After weighing, the sample was imaged with a SCANCO $\mu$-CT 80 micro-computer-tomograph (CT). During the CT measurements of approximately $2 \mathrm{~h}$, the temperature inside the measurement chamber and thus the sample was kept at $-15^{\circ} \mathrm{C}$. Immediately after the measurement, the sample was put back inside its metallic cylinder, the storage box, and the corresponding cold room. While during the weighing, the CT measurement preparation, and the imaging the isothermal environment of the samples was disturbed, we expect little effect on the microstructure at the observed resolution since these disturbances were of a very short time with respect to the snow metamorphism process timescale.

[13] The tomographic images had a nominal resolution (pixel size) of $10 \mu \mathrm{m}$, a modulation transfer function at $10 \%$ contrast level of $12.4 \mu \mathrm{m}$ and were taken at midheight of the sample cylinder. The observed region was 200 voxels ( $2 \mathrm{~mm}$ ) high. Note that, even though the imaged region of each sample was the same within $0.1 \mathrm{~mm}$ during the whole experiment, slight differences in the positioning inside the CT scanner and the densification of the snow prevented an exact one-to-one comparison or the creation of an animation of the microstructural evolution. The grayscale images (Figure 1) were filtered using a $3^{3}$ median and $5^{3}$ Gauss filter with a standard deviation of 1.2 pixels to improve the signal to noise ratio. The ice matrix of the snow was extracted by segmenting the images using a thresholding value determined by the adsorption coefficient of ice. The thresholding value was kept constant for all the segmentations during the experiment.

[14] From the measured region, a cube of $600 \times 600 \times$ 200 voxels $(6 \times 6 \times 2 \mathrm{~mm})$ was extracted, respecting the expected representative elementary volume for the snow and the properties considered [Coléou et al., 2001; Kaempfer et al., 2005]. The following structural parameters were determined:

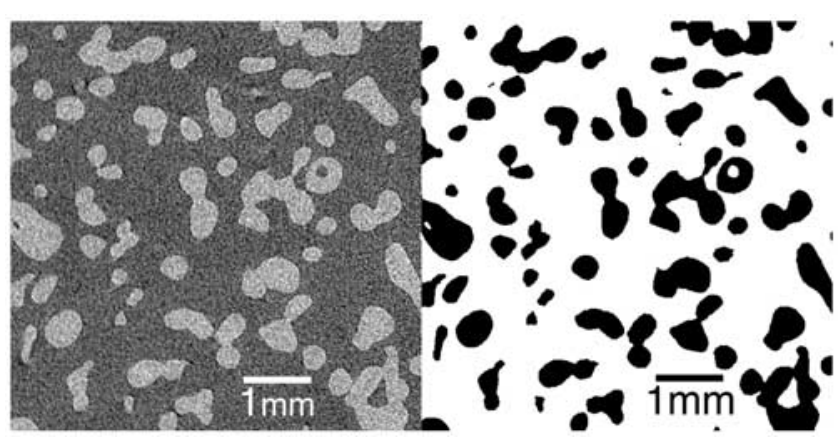

Figure 1. Unfiltered grayscale image of one tomographic slice and the corresponding final segmented image, with black corresponding to the ice. 

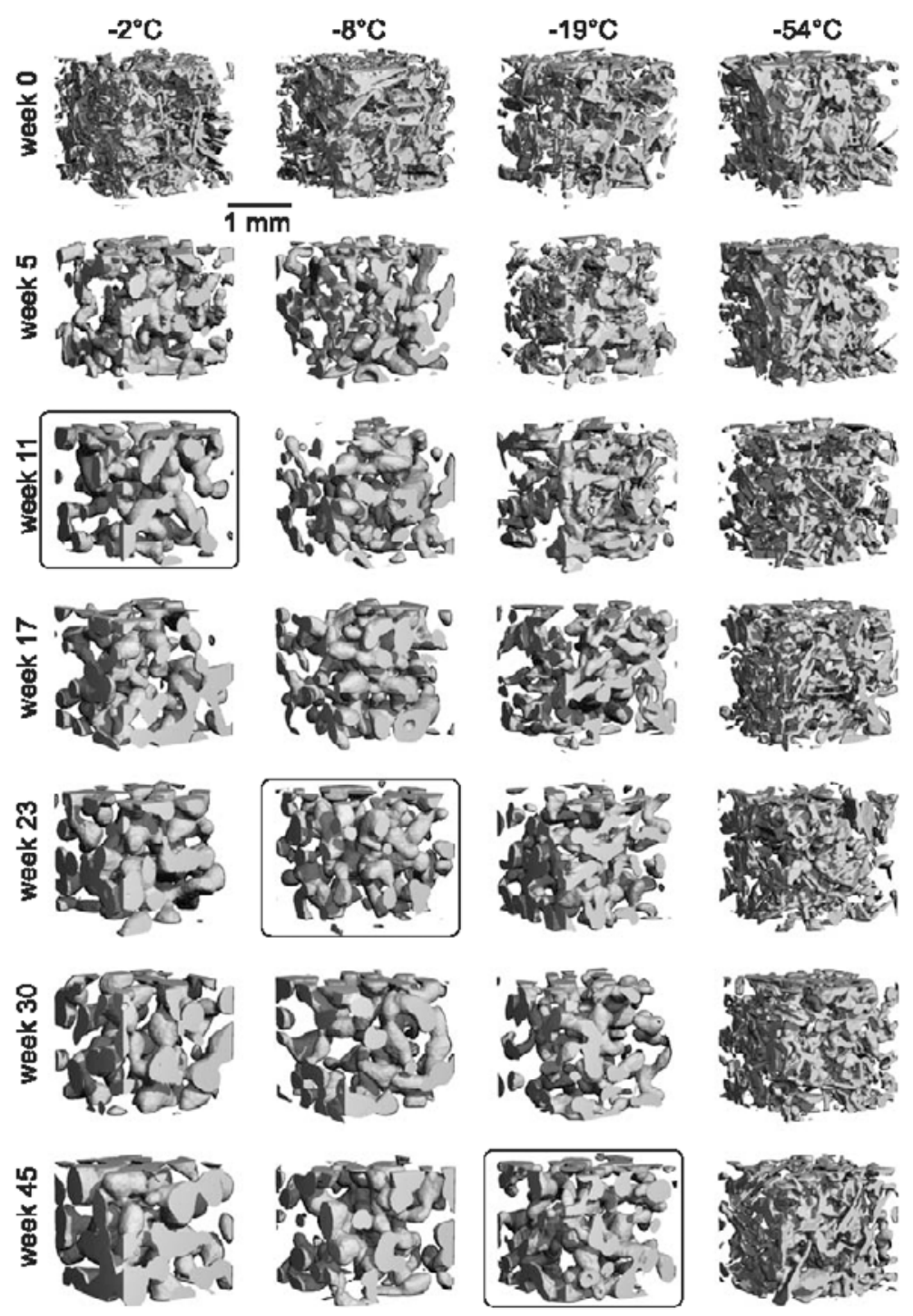

Figure 2. Evolution of the 3-D structure of the ice matrix during isothermal metamorphism. The samples 1 to 4 (from left to right) at the different measurement times from the beginning to the end (top to bottom) of the experiment. The shown cubes are $200 \times 200 \times 200$ voxels $(2 \times 2 \times 2 \mathrm{~mm})$ large. Framed images have the same specific surface area.

[15] 1. The density of the snow was calculated using tetrahedrons corresponding to the enclosed volume of the triangulated ice matrix surface, where the surface was triangulated using the marching cube method [Guilak, 1994].

[16] 2. The specific surface area (SSA) of the ice matrix was determined using the triangulated structure surface and the density as described in the paragraph above.

[17] 3. The structure model index (SMI) was computed using the normal derivative of the specific surface area, i.e., the differential variation of the SSA when the ice matrix is varied by a small amount in the direction normal to the iceair interfaces [Hildebrand and Rüegsegger, 1997b]. The SMI gives some information about the dominating form of the ice matrix. The value of the SMI would be 0,3 , and 4 for ideal plates, cylinders, and spheres, respectively.
[18] 4. The thickness histogram for the ice structure and pores was determined from the distance transform of the ice matrix and the pore space, respectively. Also, a mean thickness of the ice matrix, called trabecular thickness and noted $T b . T h$, was obtained by filling maximal spheres into the structure and taking their average size [Hildebrand and Rüegsegger, 1997a].

[19] 5. The number of traversals of ice per unit length on a linear path through the structure was called trabecular number and noted $T b . N$, given by the inverse of the mean distance between the medial axes of the ice structure [Hildebrand et al., 1999].

[20] Note that all the structural parameters considered are deduced from surface and volume measurements or the distance transform, a standard image analysis procedure. They are thus very general and do not suppose any underlying geometrical model. In particular, the terms 


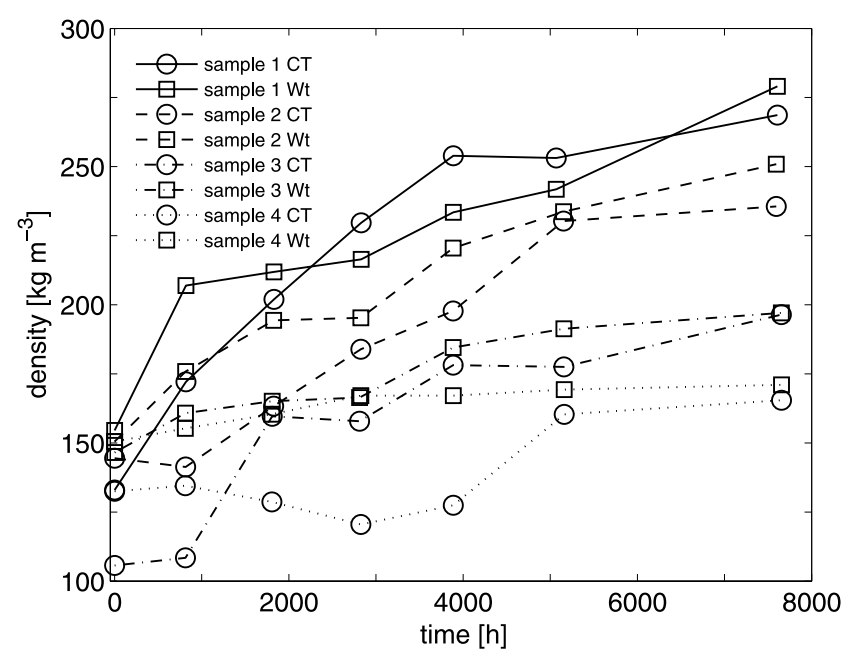

Figure 3. Evolution of the density of the snow samples. The density was computed from the CT data (circles) as well as by weighing the snow samples and measuring the height of the snow in the cylindrical sample holders to determine the volume (squares).

trabecular thickness and number do not imply a rod-like object but are merely chosen for consistency with related tomography literature.

\section{Results}

\subsection{Visual Inspection}

[21] While the structural analysis of the snow samples was conducted on the complete tomographed regions, the 3 -D evolution of the ice matrix is visually better observable on a small subregion of $200 \times 200 \times 200$ voxels $(2 \times 2 \times 2 \mathrm{~mm})$ as shown in Figure 2 . The transition from the dendritic form of the fresh snow at time zero to rounded grains a month later can be observed for the samples 1-3. At least for the samples one and two, this transition happened at an initial stage before the measurement at time 1 and is thus not resolved with our experiments. Sample 4 at the coldest temperature shows only a very slight rounding and coarsening.

\subsection{Density}

[22] One of the most important structural parameters of snow is the density. In the present work it was computed both by weighing and by image analysis of the tomographic data (Figure 3). For all the samples we note an increase in density during the whole experimental period. The density increase is low for the fourth sample which was at the lowest temperature. The discrepancies between the two density measurement techniques are suspected to be due to errors in determining the snow height in the sample cylinders due to an irregular snow surface and nonhomogeneous shrinking of the snow for the CT method. The errors however do not change the clear trend of density increase with time.

[23] The weighing of the samples showed that mass loss from sublimation was between $0.4 \%$ (sample 4 ) and $11.1 \%$ (sample 1). We do not suspect this mass loss to be critical for our analysis.

\subsection{Trabecular Thickness and Spacing}

[24] Sintering or grain growth theories frequently use parameters like grain and bond sizes. Brown et al. [1997] measured on two-dimensional sections grain and bond sizes by measuring minimal and maximal radii. This method leads to a strong bias in grain and bond size if the structures are not spherical. Because snow is rarely consisting of spherical structures, the method is questionable in most cases. The complex shapes occurring in sintered snow obscure the location of grain boundaries. We are thus forced to define other size information and chose to use the distance transform as defined by Hildebrand and Rüegsegger [1997a]. One drawback with this approach is that we gain only one thickness number for the ice matrix (and another one for the porous space) and not two distinct distributions or means as is the case with grain and bond sizes.

[25] The evolution of the histogram of the distance transform for the ice matrix and porous space for the four samples are presented in Figure 4. For better readability of the graphs, the discrete histograms, drawn only for the initial and final stage in thin lines, have been fitted by a Weibull distribution function of the form

$$
f(x)=\frac{k}{\lambda}\left(\frac{x}{\lambda}\right)^{k-1} e^{-(x / \lambda)^{k}}
$$

The values of the fitted scale parameter $\lambda$ and shape parameter $k$ are given in Tables 2 and 3 for the ice and pore histograms, respectively. The fits were mostly excellent and the $R^{2}$ always above 0.8 .

[26] We observe that for the samples 1-3 the thickness of the ice matrix increases with time during the experiment, with a faster increase at warm temperatures (sample 1) and slower increase at lower temperature (sample 3). Moreover, the thickness distribution widens with time. The comportment of the thickness of the pore space is different. While between the first and second measurement a significant increase is observed, the distribution stabilizes afterward as well with respect to its mean value as to the width of the distribution. For the fourth sample, the thickness distributions of the ice matrix and the pores hardly change.

[27] Consider now the mean value of the distance transform of the ice matrix (Figure 5) and assume that it is a similar structural information as a mean grain radius, which would be true for a material consisting of nonoverlapping spheres. Consider moreover a grain coarsening law of the form

$$
(T b . T h)^{n}-\left(T b . T h_{0}\right)^{n}=K t,
$$

where $n$ is the growth exponent and $K$ is the growth rate, as it was introduced for snow by Legagneux and Dominé [2005]. For the samples 1-3, equation (3) fits our data very well with $R^{2}>0.99$ and we obtain growth exponents of $n=$ $2.85,3.06$, and 2.10 (Figure 5). For sample 4, even though the fit is presented for completeness, the behavior of the structural evolution is quite different and $T b . T h$ changes very slowly.

\subsection{Specific Surface Area}

[28] The evolution of the specific surface area (SSA) for the four experiments is presented in Figure 6. We follow the 

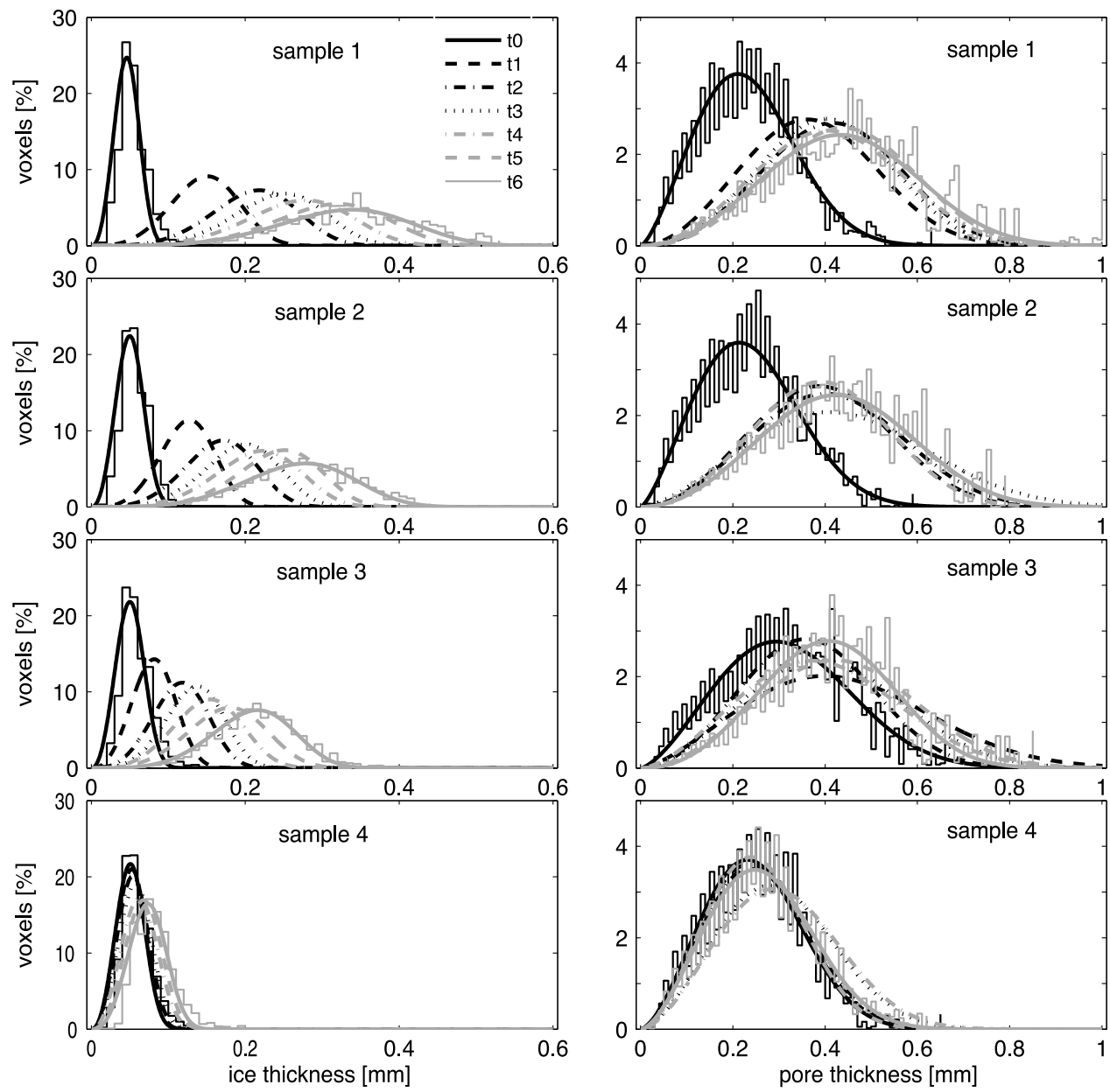

Figure 4. Evolution of the distance transform histogram of (left) the ice matrix and (right) the pore space for the samples 1-4 (top to bottom). For the times 0 and 6, the discrete histogram is plotted (thin lines), while for all the measurements only a statistical fit through the computed histogram (thick lines) is shown. Except for sample 4 where there is nearly no evolution, the ice matrix thickness increases and widens continuously with time while the pore space thickness is nearly constant after the second measurement for all the samples.

work of Legagneux et al. [2004] and Legagneux and Dominé [2005], who propose a fit of the form

$$
\operatorname{SSA}=\operatorname{SSA}_{0}\left(\frac{\tau}{\tau+t}\right)^{1 / n}
$$

Table 2. Values of the Fitted Scale and Shape Parameters $\lambda$ and $k$ of the Weibull Distribution Used to Approximate the Distance Transform Histograms of the Ice Matrix in Figure 4

\begin{tabular}{|c|c|c|c|c|c|c|c|c|}
\hline \multirow{2}{*}{$\begin{array}{l}\text { Sample } \\
\text { Number } \\
\end{array}$} & \multirow[b]{2}{*}{ Parameter } & \multicolumn{7}{|c|}{ Measurement Number, time } \\
\hline & & 0 & 1 & 2 & 3 & 4 & 5 & 6 \\
\hline 1 & $\lambda$ & 0.05 & 0.16 & 0.23 & 0.26 & 0.29 & 0.34 & $0.3 \mathrm{r}$ \\
\hline 1 & $k$ & 3.27 & 3.89 & 4.45 & 4.80 & 4.65 & 4.90 & 4.4 \\
\hline 2 & $\lambda$ & 0.06 & 0.14 & 0.18 & 0.21 & 0.24 & 0.26 & 0.2 \\
\hline 2 & $k$ & 3.23 & 4.10 & 4.23 & 4.61 & 4.59 & 5.21 & 4.4 \\
\hline 3 & $\lambda$ & 0.06 & 0.09 & 0.13 & 0.15 & 0.17 & 0.19 & 0.2 \\
\hline 3 & $k$ & 3.16 & 3.35 & 3.75 & 4.16 & 3.93 & 4.04 & 4.5 \\
\hline 4 & $\lambda$ & 0.06 & 0.06 & 0.06 & 0.06 & 0.07 & 0.08 & 0.08 \\
\hline 4 & $k$ & 3.18 & 3.18 & 3.14 & 3.17 & 3.11 & 3.31 & 3.4 \\
\hline
\end{tabular}

with $\mathrm{SSA}_{0}$ the initial SSA at time $t=0$ and $\tau$ a parameter related to the grain growth rate $K$ (see equation (3)), $\mathrm{SSA}_{0}$, and a form factor. In fact, Legagneux et al. [2004] showed that for spherical particles equations (3) and (4) are equivalent with $\tau=\frac{1}{K}\left(\frac{3}{\mathrm{SSA}_{0}}\right)^{n}$ and by introducing a constant form factor they extended the equivalence to nonspherical

Table 3. Values of the Fitted Scale and Shape Parameters $\lambda$ and $k$ of the Weibull Distribution Used to Approximate the Distance Transform Histograms of the Pore Space in Figure 4

\begin{tabular}{|c|c|c|c|c|c|c|c|c|}
\hline \multirow{2}{*}{$\begin{array}{l}\text { Sample } \\
\text { Number }\end{array}$} & \multirow[b]{2}{*}{ Parameter } & \multicolumn{7}{|c|}{ Measurement Number, time } \\
\hline & & 0 & 1 & 2 & 3 & 4 & 5 & 6 \\
\hline 1 & $\lambda$ & 0.26 & 0.42 & 0.47 & 0.45 & 0.46 & 0.49 & 0.49 \\
\hline 1 & $k$ & 2.42 & 2.93 & 3.23 & 3.22 & 2.98 & 3.27 & 3.06 \\
\hline 2 & $\lambda$ & 0.27 & 0.44 & 0.46 & 0.50 & 0.48 & 0.44 & 0.48 \\
\hline 2 & $k$ & 2.37 & 2.99 & 2.91 & 2.56 & 3.03 & 3.11 & 3.02 \\
\hline 3 & $\lambda$ & 0.36 & 0.49 & 0.41 & 0.46 & 0.45 & 0.49 & 0.46 \\
\hline 3 & $k$ & 2.47 & 2.45 & 2.96 & 2.71 & 2.62 & 2.92 & 3.27 \\
\hline 4 & $\lambda$ & 0.29 & 0.29 & 0.29 & 0.33 & 0.34 & 0.28 & 0.30 \\
\hline 4 & $k$ & 2.58 & 2.56 & 2.63 & 2.63 & 2.64 & 2.69 & 2.60 \\
\hline
\end{tabular}




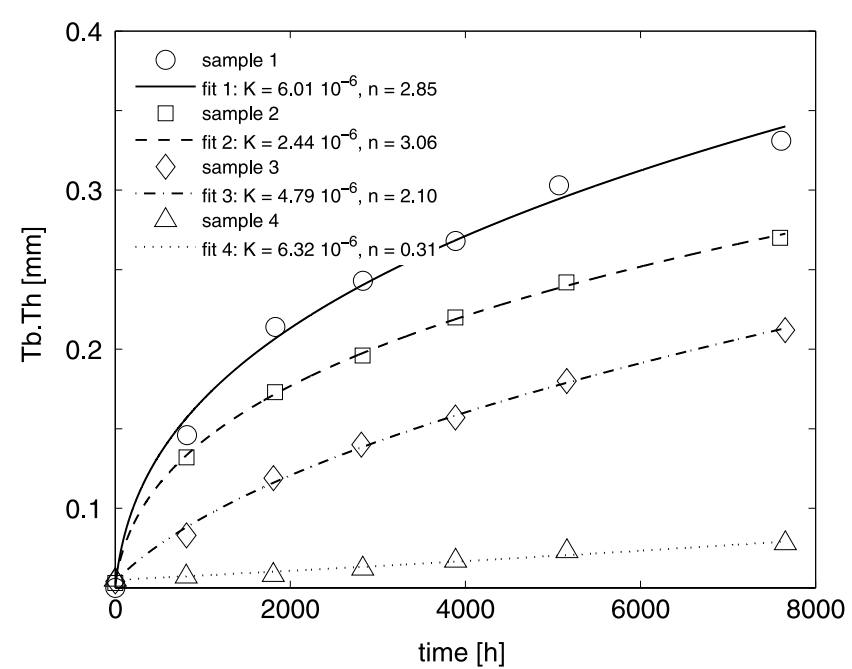

Figure 5. Evolution of the mean values of the distance transform of the ice matrix, the trabecular thickness, Tb.Th. Besides the experimental data, a fit of the form $\operatorname{Tb} \cdot \operatorname{Th}(t)=$ $\left(\left(T b . T h_{0}\right)^{n}+K t\right)^{1 / n}$ was computed.

particles. Equation (4) fits the data of the samples $1-3$ very well with $R^{2}>0.99$ and growth exponents $n$ of $3.00,3.55$, and 2.20, respectively (Figure 6). Sample 4 behaves differently and the fit is for completeness only.

\subsection{Structure Model Index and Trabecular Number}

[29] The evolution of the structure model index (SMI) of the ice matrix (Figure 7, top) confirms the visual observation of the structure evolutions. For the samples 1-3, the SMI stabilizes after an initial rapid change between the first and second measurements at values between 2.5 and 3 , the latter being the equilibrium value for cylindrical rods. For sample 4, the SMI remains constant throughout the experiment.

[30] A similar temporal trend is observed for the trabecular number Tb.N (Figure 7, bottom). At the beginning of

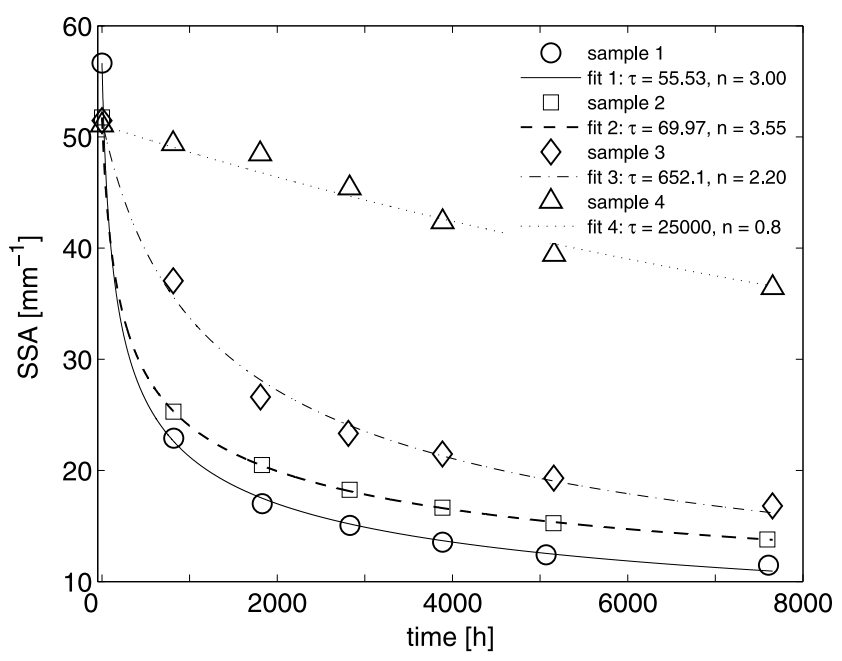

Figure 6. Evolution of the specific surface area, SSA, of the ice matrix. The computed fit is of the form $\operatorname{SSA}(t)=$ $\operatorname{SSA}_{0}\left(\frac{\tau}{\tau+t}\right)^{1 / n}$. the experiment, we expect to hit around four ice branches per millimeter unit length on a linear path through the structure, which indicates that the ice mass is finely distributed. After the first time step, the value stabilizes for the samples 1-3 around a value of two per millimeter, even though the trabecular thickness $T b . T h$ continues to evolve, as was shown above. Again, for the fourth sample no significant evolution of $T b . N$ was observed.

\section{Discussion}

[31] We consider first samples 1-3 only. From the data on the SMI and the trabecular number (Figure 7), as well as from the evolution of the histogram of the distance transform of the pore space (Figure 4, right column), the sintering process can be divided into two stages for all the three samples. Between the initial and second measurement,
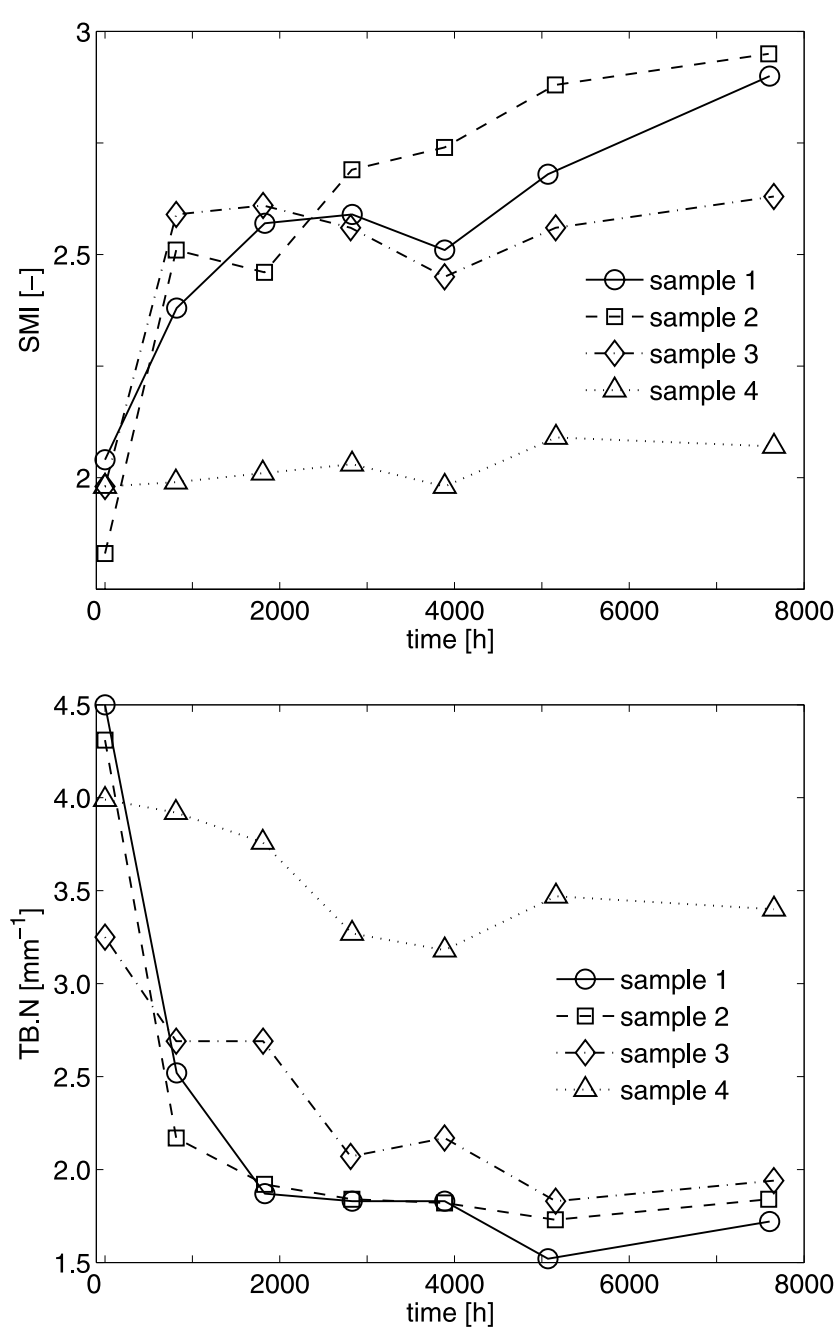

Figure 7. Evolution of (top) the structure model index (SMI) and (bottom) the trabecular number, $T b . N$ in function of time for the four samples. For the samples 1-3 the final form with an SMI close to 3 is reached during an initial stage between the first and second CT measurements, and also the trabecular number is rapidly stabilized, while for the fourth sample at the coldest temperature there is nearly no evolution of these structural parameters. 


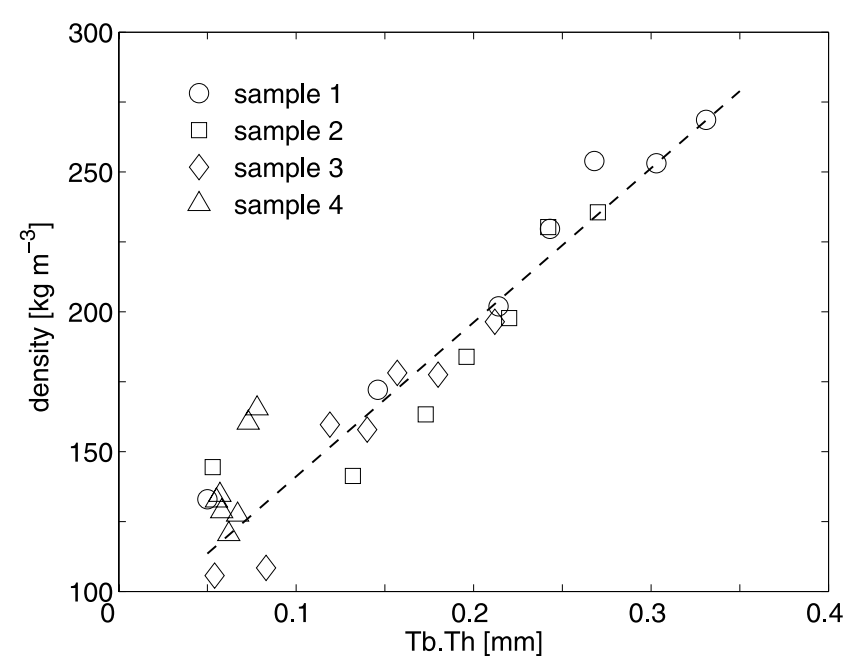

Figure 8. Evolution of the mean value of the distance transform of the ice matrix, the trabecular thickness, Tb.Th, versus the snow density. Besides the experimental data, a linear fit through the data of samples 1-3 was computed.

thus during the first month, the form of the microstructure changed toward more rounded forms as indicated by the rapid increase and following stabilization of the SMI. Simultaneously, the trabecular number decreased and the thickness of the pore space increased. In order to deduce more information about the sintering process during this initial stage, a higher temporal resolution of the microstructure observations would be needed. So far, we can conclude that a certain form of equilibrium for the microstructure is rather rapidly achieved and that this form prevails afterward, during later sintering stages. Note that the pore space thickness reaches a steady state during the first month while the ice matrix thickness increases and its distribution widens during the whole experimental period. Geometrically, this can be interpreted as that the average diameter of the air pores is constant, but the roughness of the walls, expressed here as grain diameters of ice, decreases.

[32] We observe a significant and continuous densification for all the three samples. As our snow samples were only $65 \mathrm{~mm}$ high, the stresses due to the weight are very low and densification due to creep or viscous flow can be neglected. There has thus to occur a mechanism during the isothermal sintering which leads to densification. From classical sintering theories [German, 1996] it is clear that this has to be a volume process, at least complementing surface processes, since for densification to occur, mass originating from the interior of particles must be deposited at necks. A candidate for such a volume process is grain boundary diffusion. Unfortunately, our data set does not resolve in detail the first month of sintering and the standard deviation in the density measurements prevents more detailed conclusions with respect to the initial sintering stage. However, coarsening and densification proceed hand in hand during the remainder of the experiments, as indicated by our density measurements and the thickening of the ice matrix at nearly constant pore spacing. That coarsening and densification are indeed related becomes evident when we compare the evolutions of the density and trabecular thick- ness (Figure 8): The two structural parameters relate linearly. Moreover, the slope of the linear relationship is independent of temperature, implying that the density solely depends on grain size, which is represented here by the trabecular thickness. This is consistent with several observations in powder compacts [Gupta, 1972; Bruch, 1962]. Lange and Kellett [1989] used a model for the sintering of spherical particles of different sizes to show that grain growth within a sintering array can be controlled by coarsening kinetics. They showed that after a grain disappears from between larger grains because of coarsening, a new nonequilibrium develops between the grain boundary and the surface free energies for the newly joined grains; the driving force for sintering is reinitiated. Thus grain growth via coarsening can continually drive sintering and array shrinkage. They found that, once the initial particles sinter together, further densification is limited by coarsening kinetics and confirmed a nearly linear relation between grain size and density. Our data are consistent with this reasoning.

[33] The evolution of the trabecular thickness and the SSA of the ice matrix follows very well a logarithmic trend. We analyze this evolution on the basis of the theories developed by Legagneux et al. [2004] and Legagneux and Dominé [2005], who developed a model for the SSA decrease during isothermal metamorphism based on the physical processes involved in Ostwald ripening. The driving force in this model is the reduction of the SSA and an important model hypothesis is that mass transfer occurs by sublimation, transport through the gas phase, and resublimation. The model is characterized by the growth law given by equation (3). Theoretically, the growth exponent $n$ is 2 if the surface processes and 3 if the diffusion step are rate limiting. Best fits through our data set lead to values of $n=$ $2.85,3.06$, and 2.10 (see Figure 5) for samples $1-3$. Under some model hypothesis, in particular that the form of the considered particles is time-independent, Legagneux et al. [2004] transformed the growth law (equation (3)) into an evolution equation for the SSA (equation (4)). The fit to the SSA data of our experiments leads to values of $n=3.00$, 3.55 , and 2.20 for the samples $1-3$. The fit is excellent $\left(R^{2}>\right.$ $0.99)$ for all the samples which suggests that the SSA decreases indeed logarithmically with time during isothermal snow metamorphism. However, the model hypothesis of a time-independent particle form is clearly violated at the beginning of our experiments and it is thus not surprising to find different growth exponents than the ones based on the grain growth law of equation (3). However, the discrepancies between the two models do not diminish if we reduce our analysis to the second stage of the sintering process with quasi constant grain forms as characterized by the SMI and visually observed to be mostly rounded. Second stage growth exponents for both models, computed by omitting the first and the first two measurements, are summarized in Table 4. While the model discrepancies remain, we find the expected trend of a decreasing growth exponent from warmer temperatures (sample 1) to colder temperatures (sample 3) for the restricted data set of times 2 to 6 . So far, we cannot determine if the discrepancies between the theory and the experimental growth exponents are due to the simultaneous presence of volume diffusion phenomena or the violation of the model hypotheses. In particular, for 
Table 4. Growth Exponents for the Tb.Th Growth Law (Equation (3)) and the Relation for the SSA Decrease (Equation (4)) for a Best Fit Using All the Data Points (Times 0-6), All but the First (Times 1-6), and All but the First and Second (Times 2-6) Measurements

\begin{tabular}{|c|c|c|c|c|c|c|}
\hline \multirow{2}{*}{$\begin{array}{l}\text { Sample } \\
\text { Number }\end{array}$} & \multicolumn{2}{|c|}{ Times $0-6$} & \multicolumn{2}{|c|}{ Times $1-6$} & \multicolumn{2}{|c|}{ Times $2-6$} \\
\hline & Tb.Th & $S S A$ & $T b . T h$ & $S S A$ & $T b . T h$ & $S S A$ \\
\hline 1 & 2.85 & 3.00 & 3.65 & 3.92 & 3.37 & 4.05 \\
\hline 2 & 3.06 & 3.55 & 3.21 & 3.40 & 3.18 & 3.35 \\
\hline 3 & 2.10 & 2.20 & 2.46 & 3.57 & 2.00 & 2.39 \\
\hline 4 & 0.31 & 0.80 & 0.95 & 0.24 & 3.68 & 3.10 \\
\hline
\end{tabular}

the grain growth law (equation (3)), the hypothesis of nonoverlapping particles is violated in snow with its connected ice matrix and, for the equivalence between equations (3) and (4), the hypothesis of a constant grain form might not be respected sufficiently well even for the second sintering stage. Consequently, an interpretation of the growth exponents with respect to the physical processes seems ambiguous. Nevertheless, the logarithmic SSA decrease remains and our data suggests that a sintering model where the reduction of the SSA is rate limiting is quite accurate for long-term isothermal snow metamorphism.

[34] While our experiments seem thus to indicate that coarsening is the limiting process during long-term isothermal snow metamorphism, the results also indicate the continuous presence of a volumetric diffusion process which leads to densification. From the structural data gathered and analyzed so far, it seems difficult to identify which process this would be. Colbeck [1997, 1998, 2001] proposes, consistent with the fact that ice is a crystalline material, grain boundary diffusion as an important process during isothermal sintering of ice. Since grain boundaries are not directly observable by X-ray tomography, we lack any bond size information which would allow us to test this theory. One experimental tack could be to determine the evolution of the dihedral angle between ice grains and relate it to the theoretical evolution presented by Colbeck [1998]. Grain boundary grooves are clearly visible in the tomography images for the more rounded forms from times 1 to 6 , as for sample 1 after 45 weeks (Figure 9) and it should be possible to measure dihedral angles. However, a relatively fast evolution toward the equilibrium dihedral angle of about $145^{\circ}$ [Colbeck, 1998] would be expected and we would need a higher resolution in time during the first month. Moreover, an algorithm for the determination of the dihedral angle also at early stages of the sintering process would have to be developed. Another problem when trying to relate the structural information to the theory could be that most models for grain boundary diffusion are very rudimentary and consider sintering of spherical particles only or are limited to two interacting grains compared to the complex multigrain system we encounter in snow.

[35] Finally, we discuss sample 4, kept at a temperature of $-54^{\circ} \mathrm{C}$. This sample shows a distinctively different evolution than the other three samples. During the observed 11 month period, there is very little change in the microstructure of snow at this low temperature. While the SMI stayed quasi constant, there was a slight decrease of the trabecular number, an increase of the trabecular thickness that is well fitted $\left(R^{2}=0.97\right)$ by a linear regression with slope $3.210^{-6}$ and the SSA decreased slowly by only $19 \%$. The SSA decrease is also well fitted linearly with a slope of -0.002 and $R^{2}=0.97$. The densification was less than $10 \%$. Snow metamorphism at $-54^{\circ} \mathrm{C}$ was thus very slow and we suspect that the temperature being below the point of homogenous nucleation of water $\left(-42^{\circ} \mathrm{C}\right)$ or the absence of the quasi-liquid layer at these temperatures is at the cause of the much slower sintering. These very low metamorphic changes might have important implications for the densification of firn in the isothermal zone of Antarctic snowpacks.

\section{Conclusion}

[36] Four isothermal metamorphism experiments of snow have been performed during nearly 1 year at temperatures occurring in the alpine and polar environment. Approximately every $40 \mathrm{~d}$, the snow microstructure was observed by X-ray microtomography and structural parameters were deduced. The data have been analyzed using ice sintering theories.

[37] At the coldest temperature of $-54^{\circ} \mathrm{C}$, the obtained data shows almost no sign of snow metamorphism. Only very small changes in the microstructure were observed, in particular the SSA of the ice matrix decreased by only $19 \%$ during 1 year, while parameters like the SMI stayed quasi constant and virtually no shape change and rounding could be observed.

[38] For all the other samples $(1-3)$ at warmer temperatures, the evolution of the form parameter SMI, the trabecular number, and the histogram of the distance transform of the pore space can be interpreted as two stages in the sintering process. Before the second structural measurement, the ice matrix approached a rounded form, while the trabecular number decreased from approximately 4.5 to $2.5 \mathrm{~mm}^{-1}$. From the second measurement on, the SMI, the trabecular number, and the pore space distance transform did not evolve anymore; a certain structural equilibrium was reached.

[39] Continuous densification was observed for the samples $1-3$. The stresses due to weight being negligible, this is a clear indication that volumetric sintering processes have to occur. Moreover, the density and the ice matrix thickness (trabecular thickness) related linearly to one another, which indicates that the same physical process limits both densification and coarsening. The linear relationship was independent of the sintering temperature.

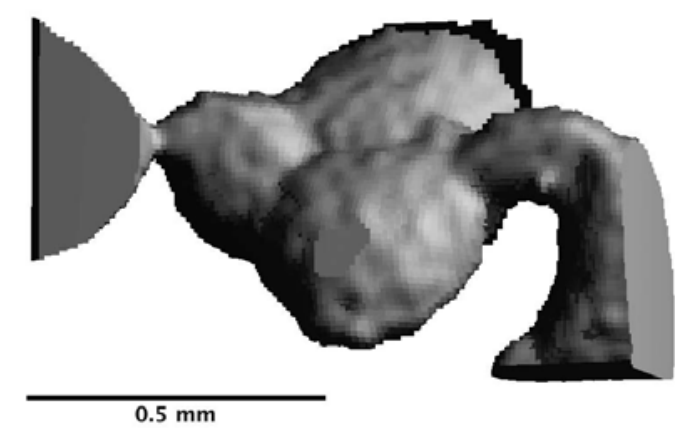

Figure 9. Detailed view of the three-dimensional snow structure of sample 1 at scan time 45 weeks showing grain boundaries with triple-junction in the center of one "snow grain" and bonds connecting to two neighboring grains 
[40] Except for the sample at the coldest temperature, the increase with time of the trabecular thickness and a corresponding decrease in the SSA of the ice matrix are very well fitted by a power law. This suggests that coarsening is the rate limiting process. In the past, such an evolution was associated with a theory based on Ostwald ripening and a dominating process of evaporation-condensation [Legagneux et al., 2004]. There are however discrepancies between our experimental growth exponents and this theory. This might be due to the complex coupling of different diffusion mechanisms during snow metamorphism but also due to violated model hypotheses. While our 3-D parameters proved to be very useful to describe and measure structural properties of metamorphosing snow with a wide size distribution, more structural information, ideally related to the grain boundaries, would be needed in order to confirm whether the volumetric process leading to densification is grain boundary diffusion. Moreover, to analyze the first stage of sintering a higher temporal resolution would be needed.

[41] Acknowledgments. Daniela Schmid operated the micro-CT, and Bernd Pinzer modeled the effect of temperature disturbances on the sample. We thank Sergey Sokratov (Moscow State University) for his helpful comments on a preliminary draft and three anonymous reviewers for their constructive suggestions that helped improve the manuscript. The research was supported by the Swiss National Science Foundation grant 200021100294 .

\section{References}

Arnaud, L., V. Lipenkov, J. Barnola, M. Gay, and P. Duval (1998), Modelling of the densification of polar firn: Characterization of the snow-firn transition, Ann. Glaciol., 26, 39-44.

Arons, E. M., and S. C. Colbeck (1995), Geometry of heat and mass transfer in dry snow: A review of theory and experiment, Rev. Geophys., 33(4), 463-493.

Brown, R. L., M. Q. Edens, M. Barber, and A. Sato (1997), Equitemperature metamorphism of snow, in Snow Engineering: Recent Advances (Proceedings of the Third International Conference on Snow Engineering, Sendai, Japan, 26-31 May, 1996), edited by M. Izumi, T. Nakamura, and R. L. Sack, pp. 41-48, A. A.Balkema, Rotterdam, Netherlands.

Bruch, C. A. (1962), Sintering kinetics for the high density alumina process, Am. Ceram. Soc. Bull., 41(12), 799-806.

Cabanes, A., L. Legagneux, and F. Dominé (2003), Rate of evolution of the specific surface area of surface snow layers, Environ. Sci. Technol., 37(4), 661-665, doi:10.1021/es025880r.

Colbeck, S. C. (1997), A review of sintering in seasonal snow, Tech. Rep. 97-10, Cold Reg. Res. and Eng. Lab., Hanover, N. H.

Colbeck, S. C. (1998), Sintering in a dry snow cover, J. Appl. Phys., 84(8), 4585-4589, doi:10.1063/1.368684.

Colbeck, S. C. (2001), Sintering of unequal grains, J. Appl. Phys., 89(8), 4612-4618, doi:10.1063/1.1356427.

Coléou, C., B. Lesaffre, J.-B. Brzoska, W. Ludwig, and E. Boller (2001), Three-dimensional snow images by X-ray microtomography, Ann. Glaciol., 32, 75-81.

Dominé, F., and P. Shepson (2002), Air-snow interactions and atmospheric chemistry, Science, 297(5586), 1506-1510, doi:10.1126/science.1074610.

Flanner, M. G., and C. S. Zender (2006), Linking snowpack microphysics and albedo evolution, J. Geophys. Res., 111, D12208, doi:10.1029/ 2005JD006834.

Flin, F., J. B. Brzoska, B. Lesaffre, C. Coléou, and R. A. Pieritz (2003), Full three-dimensional modelling of curvature-dependent snow metamorphism: First results and comparison with experimental tomographic data, J. Phys. D, 36(10A), A49-A54, doi:10.1088/0022-3727/36/10A/310.

Flin, F., J. B. Brzoska, B. Lesaffre, C. Coléou, and R. A. Pieritz (2004), Three-dimensional geometric measurements of snow microstructural evolution under isothermal conditions, Ann. Glaciol., 38, 39-44.
German, R. M. (1996), Sintering Theory and Practice, John Wiley, New York.

Guilak, F. (1994), Volume and surface area of viable chondrocytes in situ using geometric modelling of serial confocal sections, J. Microsc., 173, $245-256$.

Gupta, T. K. (1972), Possible correlation between density and grain size during sintering, J. Am. Ceram. Soc., 55(5), 276-277.

Hildebrand, T., and P. Rüegsegger (1997a), A new method for the modelindependent assessment of thickness in three-dimensional images, $J . M i-$ crosc., 185(1), 67-75, doi:10.1046/j.1365-2818.1997.1340694.x.

Hildebrand, T., and P. Rüegsegger (1997b), Quantification of bone microarchitecture with the structure model index, Comput. Methods Biomech. Biomed. Eng., 1, 15-23.

Hildebrand, T., A. Laib, R. Müller, J. Dequeker, and P. Rüegsegger (1999), Direct three-dimensional morphometric analysis of human cancellous bone: Microstructural data from spine, femur, iliac crest, and calcaneus, J. Bone Miner. Res., 14(7), 1167-1174, doi:10.1359/jbmr.1999.14.7. 1167.

Hobbs, P. V., and B. J. Mason (1963), The sintering and adhesion of ice, Philos. Mag., 9, 181-197.

Kaempfer, T. U., M. Schneebeli, and S. A. Sokratov (2005), A microstructural approach to model heat transfer in snow, Geophys. Res. Lett., 32, L21503, doi:10.1029/2005GL023873.

Kingery, W. D. (1960), Regelation, surface diffusion, and ice sintering, J. Appl. Phys., 31(5), 833-838.

Kuroiwa, D. (1961), A study of ice sintering, Tellus, 13(2), 252-259.

Kuroiwa, D. (1962), A study of ice sintering, Tech. Rep. 86, Cold Reg. Res. and Eng. Lab., Hanover, N. H.

Lange, F. F., and B. J. Kellett (1989), Thermodynamics of densification: II, Grain growth in porous compacts and relation to densification, $J$. Am Ceram. Soc., 72(5), 735-741.

Legagneux, L., and F. Dominé (2005), A mean field model of the decrease of the specific surface area of dry snow during isothermal metamorphism, J. Geophys. Res., 110, F04011, doi:10.1029/2004JF000181.

Legagneux, L., T. Lauzier, F. Dominé, W. F. Kuhs, T. Heinrichs, and $\mathrm{K}$. Techmer (2003), Rate of decay of specific surface area of snow during isothermal experiments and morphological changes studied by scanning electron microscopy, Can. J. Phys., 81(1-2), 459-468.

Legagneux, L., A. S. Taillandier, and F. Dominé (2004), Grain growth theories and the isothermal evolution of the specific surface area of snow, J. Appl. Phys., 95(11), 6175-6184, doi:10.1063/1.1710718.

Legrand, M., and P. Mayewski (1997), Glaciochemistry of polar ice cores: A review, Rev. Geophys., 35(3), 219-243.

Maeno, N., and T. Ebinuma (1983), Pressure sintering of ice and its implication to the densification of snow at polar glaciers and ice sheets, J. Phys. Chem., 87(21), 4103-4110.

Rosenthal, W., J. Saleta, and J. Dozier (2006), Scanning electron microscopy of impurity structures in snow, Cold Reg. Sci. Technol., 47, 80-89, doi:10.1016/j.cold.regions.2006.08.006.

Schweizer, J., J. B. Jamieson, and M. Schneebeli (2003), Snow avalanche formation, Rev. Geophys., 41(4), 1016, doi:10.1029/2002RG000123.

Shapiro, L. H., J. B. Johnson, M. Sturm, and G. L. Blaisdell (1997), Snow mechanics-Review of state of knowledge and applications, Tech. Rep. 97-3, Cold Reg. Res. and Eng. Lab., Hanover, N. H.

Sokratov, S. A., and R. G. Barry (2002a), Intraseasonal variation in the thermoinsulation effect of snow cover on soil temperatures and energy balance, J. Geophys. Res., 107(D10), 4093, doi:10.1029/2001JD000489.

Sokratov, S. A., and R. G. Barry (2002b), Correction to "Intraseasonal variation in the thermoinsulation effect of snow cover on soil temperatures and energy balance," J. Geophys. Res., 107(D19), 4374, doi:10.1029/ 2002JD001595

Taillandier, A.-S., F. Domine, W. R. Simpson, M. Sturm, and T. A. Douglas (2007), Rate of decrease of the specific surface area of dry snow: Isothermal and temperature gradient conditions, J. Geophys. Res., 112, F03003, doi:10.1029/2006JF000514

Warren, S. G. (1982), Optical properties of snow, Rev. Geophys., 20(1), $67-89$.

T. U. Kaempfer, Cold Regions Research and Engineering Laboratory, 72 Lyme Road, Hanover, NH 03755, USA. (thomas.kaempfer@erdc.usace. army.mil)

M. Schneebeli, WSL Swiss Federal Institute for Snow and Avalanche Research, Flüelastrasse 11, CH-7260 Davos, Switzerland. (schneebeli@ slf.ch) 